\title{
2019 Athanasiou ABME Student Awards
}

\author{
(Published online 25 November 2019)
}

The Kyriacos and Kiley Athanasiou Endowment was established in 2017 within the Biomedical Engineering Society (BMES) to promote biomedical engineering scholarship and honor the contributions of Dr. Kyriacos Athanasiou to the field. One goal of the endowment is to recognize excellence in future leaders of biomedical engineering by awarding the top papers published in the Annals of Biomedical Engineering (ABME) by graduate students and post-doctoral scholars. The second Athanasiou ABME Student Award Session was held at the 2019 BMES Annual Meeting. Six awardees were selected, five of which were able to attend BMES to present their papers at the session (Fig. 1).

The Athanasiou ABME Student Awards were selected from all papers with graduate student or postdoctoral scholar first authors that were published online between April 2018 and March 2019. There were a total of 102 papers that met the criteria, with 83 written by graduate students and 19 written by post-doctoral scholars. The number and rate of citations and downloads, along with the impact and quality of the papers were used to select the awardees. Each deputy editor-in-chief selected six finalists, from which the editor-in-chief selected the award winners. Two of the papers had two co-first authors, so there were eight total awardees. Following the inaugural student award session in 2018, there are now a total of 14 Athanasiou awardees. $^{1,2,7,8,10,12}$ The first author from each paper was contacted to notify them and ask them to present their work at the award session. The papers covered a wide range of topics including biomaterials, biomechanics, and medical and therapeutic devices.

Manuchehrabadi et al. developed a new method for warming vitrified biomaterials. ${ }^{11}$ Materials for tissue transplantation can be preserved in a vitrified state, but successful rewarming is often a challenge. This new method used inductive warming with commercially available metals, and maintained high viability in a vitrified carotid artery after rewarming.

Kalli et al. evaluated the effects of compressive forces on pancreatic fibroblasts. ${ }^{9}$ These cells play an important role in pancreatic cancer progression, and can produce excess extracellular matrix that contributes to increased compressive forces within the tumor. The results of this study showed that com- pressive stress stimulates fibroblast activation and promotes cancer cell migration. A better understanding of the mechanisms behind these findings could lead to new therapeutic interventions.

Dixon et al. evaluated the efficacy of large diameter microbubbles for use in sonothrombolysis. ${ }^{5}$ This clot dissolution technique normally uses commercially available microbubbles that are smaller diameter and have longer lifetimes, but the larger diameter bubbles showed promise for enhancing thrombolysis rates.

Chang et al. developed a semi-passive rehabilitation robot for patients with neurologic or orthopedic disorders. ${ }^{3}$ The device was validated through theoretical analyses, hardware experiments, and human subject testing, and showed potential for use as a low-cost therapeutic tool for upper-extremity rehabilitation.

Cheng et al. presented a new method for detecting venous entry during peripheral intravenous catheterization. ${ }^{4}$ The method uses a concentric electrode needle that measures bio-impedance of the contacting tissue. An indicator light identifies when the measured value is within the range of blood. An animal model was used to demonstrate the accuracy of the device for detecting blood bio-impedance, while naïve subjects tested the device on a baby arm phantom to show the advantage in performance it provided.

Gabler et al. developed a new brain injury metric to predict brain strain from head kinematics during an impact. ${ }^{6}$ The metric is based on the deformation response of a second-order mechanical system, and calculates strain-based responses from input angular velocity and acceleration. The metric was highly correlated with strain responses output by finite element models for the same impact conditions.

The next Athanasiou ABME Student Award Session will be held at the 2020 BMES Annual Meeting in San Diego (Fig. 2). Papers with graduate student or post-doctoral scholar first authors that were published during 2019 will be considered for the award. Awardees will be notified by May 1, 2020. Each awardee will receive a $\$ 500$ honorarium and free meeting registration, and will be asked to present their paper at the award session. We look forward to another excellent session showcasing the best graduate student and post-doctoral scholar papers published in ABME. 


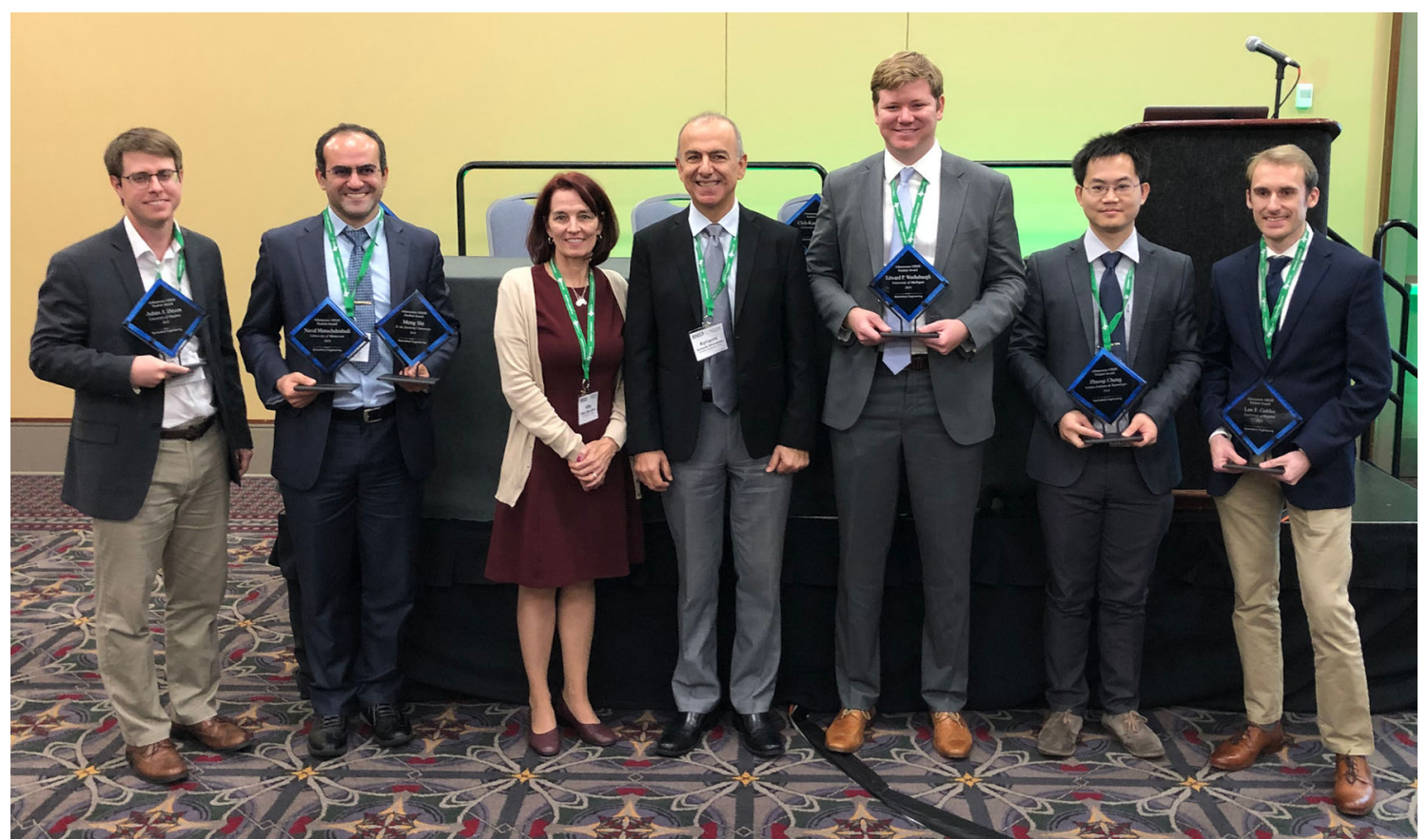

FIGURE 1. 2019 Athanasiou ABME Student Award session. Pictured left to right: Adam Dixon, Navid Manuchehrabadi, Kiley Athanasiou, Kyriacos Athanasiou, Edward Washabaugh, Zhuoqi Cheng, and Lee Gabler.

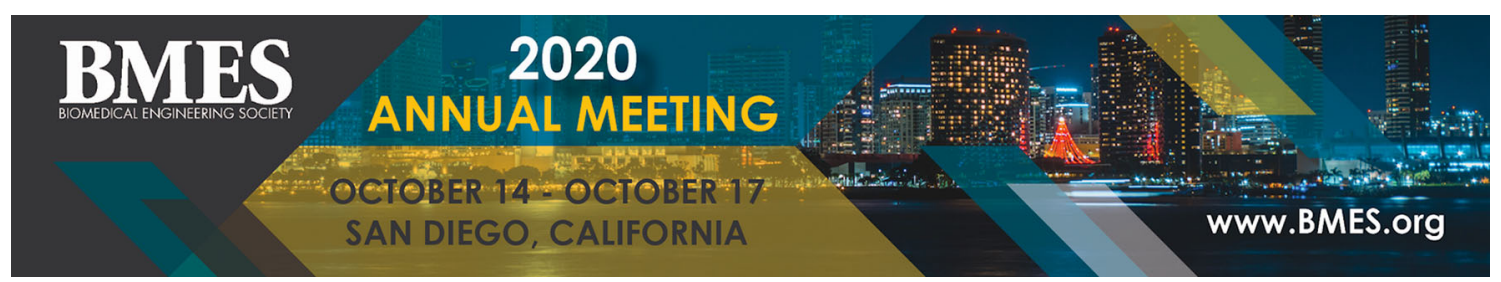

FIGURE 2. Join us for the next Athanasiou Awards Session at the 2020 BMES Annual Meeting in San Diego.

\section{REFERENCES}

${ }^{1}$ Campolettano, E. T., M. L. Bland, R. A. Gellner, D. W. Sproule, B. Rowson, A. M. Tyson, S. M. Duma, and S. Rowson. Ranges of injury risk associated with impact from unmanned aircraft systems. Ann. Biomed. Eng. 45:27332741, 2017.

${ }^{2}$ Chan, W.-Y., J. Yip, K.-L. Yick, S.-P. Ng, L. Lu, K. M.-C. Cheung, K. Y.-H. Kwan, J. P.-Y. Cheung, K. W.-K. Yeung, and C.-Y. Tse. Mechanical and clinical evaluation of a shape memory alloy and conventional struts in a flexible scoliotic brace. Ann. Biomed. Eng. 46:1194-1205, 2018.

${ }^{3}$ Chang, C.-K., E. P. Washabaugh, A. Gwozdziowski, C. D. Remy, and C. Krishnan. A semi-passive planar manipulandum for upper-extremity rehabilitation. Ann. Biomed. Eng. 46:1047-1065, 2018.

${ }^{4}$ Cheng, Z., B. L. Davies, D. G. Caldwell, and L. S. Mattos. A new venous entry detection method based on electrical bio-impedance sensing. Ann. Biomed. Eng. 46:1558-1567, 2018.

${ }^{5}$ Dixon, A. J., J. M. R. Rickel, B. D. Shin, A. L. Klibanov, and J. A. Hossack. In vitro sonothrombolysis enhancement by transiently stable microbubbles produced by a flow-fo- cusing microfluidic device. Ann. Biomed. Eng. 46:222-232, 2018

${ }^{6}$ Gabler, L. F., J. R. Crandall, and M. B. Panzer. Development of a metric for predicting brain strain responses using head kinematics. Ann. Biomed. Eng. 46:972-985, 2018.

${ }^{7}$ Horeman, T., E. Buiter, B. Pouran, M. Stijntjes, J. Dankelman, and G. Tuijthof. In-vitro detection of small isolated cartilage defects: intravascular ultrasound vs. optical coherence tomography. Ann. Biomed. Eng. 46:1745-1755, 2018.

${ }^{8}$ Horvath, M. A., C. E. Varela, E. B. Dolan, W. Whyte, D. S. Monahan, C. J. Payne, I. A. Wamala, N. V. Vasilyev, F. A. Pigula, and D. J. Mooney. Towards alternative approaches for coupling of a soft robotic sleeve to the heart. Ann. Biomed. Eng. 46:1534-1547, 2018.

${ }^{9}$ Kalli, M., P. Papageorgis, V. Gkretsi, and T. Stylianopoulos. Solid stress facilitates fibroblasts activation to promote pancreatic cancer cell migration. Ann. Biomed. Eng. 46:657-669, 2018.

${ }^{10}$ Liu, H., E. Auvinet, J. Giles, and F. Rodriguez y Baena. Augmented reality based navigation for computer assisted 
hip resurfacing: a proof of concept study. Ann. Biomed. Eng. 46:1595-1605, 2018.

${ }^{11}$ Manuchehrabadi, N., M. Shi, P. Roy, Z. Han, J. Qiu, F. $\mathrm{Xu}, \mathrm{T}$. J. Lu, and J. Bischof. Ultrarapid inductive rewarming of vitrified biomaterials with thin metal forms. Ann. Biomed. Eng. 46:1857-1869, 2018.

${ }^{12}$ Singh, H., H. N. Modi, S. Ranjan, J. W. Dilley, D. Airantzis, G.-Z. Yang, A. Darzi, and D. R. Leff. Robotic surgery improves technical performance and enhances prefrontal activation during high temporal demand. Ann. Biomed. Eng. 46:1621-1636, 2018.

\section{BETHANY ROWSON}

Department of Biomedical Engineering and Mechanics Virginia Tech, Blacksburg, VA

Electronic mail: browson@vt.edu

Publisher's Note Springer Nature remains neutral with regard to jurisdictional claims in published maps and institutional affiliations. 